\title{
Real-Time UML
}

\author{
Bruce Powel Douglass
}

I-Logix

\begin{abstract}
The UML (Unified Modeling Language) is a third-generation object-oriented modeling language recently accepted as a standard by the OMG (Object Management Group). The OMG is an association of over 800 leading technology companies that have banded together to promote standards in the object community. The UML standard draws on the last decade of intensive experience in object modeling, discarding features that didn't work and enhancing support for those that have proven their value. The UML has successfully been applied to the development of real-time and embedded systems in many different vertical markets from medical systems to avionics to military systems to office automation. The UML is clearly and demonstrably completely adequate to model and develop real-time systems without requiring extensions or modifications. By "Real-Time UML", I mean the application of the UML standard to the development of real-time and embedded systems, and focusing attention on those aspects of UML especially relevant to the areas of special concern for such systems.
\end{abstract}

\section{Real-Time Issues}

Real-time systems have all the problems of "normal" software systems, such as capturing requirements, designing functionality, proving correctness, and testing, plus several more: timeliness, concurrency, predictability, efficiency, distribution and communication, fault tolerance (and related issues of reliability and safety), and hardware interfacing. Standard structured methods don't deal explicitly with any of these issues. Is the UML any better? The answer, as it turns out, is "YES!" Let's deal with each of these issues in turn.

\subsection{Timeliness}

The timeliness of an action is most often modeled as the completion of the action with respect to a deadline. Deadlines may be "hard" or "soft". Missing a hard deadline constitutes a system failure of some kind, so great care must be taken to ensure that all such actions execute in a timely way. The important modeling concerns of timeliness are modeling execution time, deadlines, arrival patterns, synchronization patterns (see concurrency, below) and time sources.

Timeliness requirements may be addressed by first determining the end-toend performance requirement of an event-response action sequence. These are normally determined during use case or context analysis. For example, a deadline 
might exist from an external perspective: e.g. "when the actor VolumeControl sends an Increase command, the system shall respond within $10 \mathrm{~ms}+/-2 \mathrm{~ms} . "$ Both use case and context analysis view the system from a black-box perspective and serve as a means of capturing external requirements, such as overall performance and response times. Once the "box is opened" and classes are identified, a sequence diagram shows the objects (and operations) involved in handling the request and controlling the output response. Each operation in the sequence is given a portion of that budget such that the sum of all execution times for each operation in the sequence, including potential blocking, is less than or equal to the overall performance budget. These budgets may be captured as constraints associated with the class operations or captured graphically on sequence diagrams using timing constraint expressions.

Time sources themselves are modeling implicitly using the $\operatorname{tm}()$ operator defined for statecharts, which specifies the precondition for the transition is that a fixed period of time has elapsed. It is also simple to explicitly model OS and hardware timers as classes that propagate timeout events to client objects.

\subsection{Concurrency}

Concurrency is the simultaneous (or non-deterministic interleaved) execution of multiple sequential chains of actions. These chains may execute on one processor ("pseudoconcurrency") or multiple processors ("true concurrency"). The UML models concurrency by identifying a small set of "active" objects within a system. These active objects are the roots of the system's concurrent threads. Active objects are similar to Ada's task types but have all the power of normal classes as well. Idiomatically, it is common to construct a "task diagram" by simply including only the active objects on a single class diagram. Often, active objects are composite objects that tightly aggregate their component parts that, in turn, execute within the thread of their composite.

One of the issues with concurrent reactive systems is the arrival patterns of the initiating events. Two main categories are periodic and aperiodic. A periodic arrival pattern means that the thread is re-initiated on a fixed period, plus or minus a small variation (jitter). Such threads are commonly called periodic tasks. Aperiodic tasks don't occur with a regular period. Instead, they appear to occur (to the system, at least) randomly. For such systems to be schedulable, the frequency of occurrence of the initiating event must be bounded in time. One such bound is a minimum interarrival time, that is, a minimum time that must elapse between incoming events. A minimum interarrival bound is normally used when the response deadline is "hard". Another common aperiodic bound is an average event rate. This is used with so-called "soft" deadlines.

If concurrent threads were truly independent, life would be much less interesting. Fortunately (or unfortunately, depending on your outlook), threads must communicate and share resources. Communication in object systems takes place via messages. Messaging is a logical abstraction that includes a variety of rendezvous patterns, including synchronous function calls, asynchronous, waiting, timed, and balking. By far, the most common are synchronous function calls. 
Objects in one thread directly call the methods of objects in another, effectively operating within the same thread of control. Asynchronous calls are supported by real-time operating systems by queuing a message from another thread and acting on it when it is convenient, but meanwhile the caller continues without waiting. A waiting rendezvous means that the calling task will wait indefinitely for the called task to become ready, while in a timed rendezvous the caller will wait for a specified length of time before aborting the synchronization attempt. Finally, a balking rendezvous means simply that if the called task is not immediately available, the caller will abort the attempt and take other actions.

Rendezvous are specified within object methods by stereotyping messages and events with a rendezvous pattern. Some common message stereotypes are identified in [4. It is important to characterize the rendezvous patterns of crossthread messaging in order to ensure deadlines will always be met.

Another common problem in concurrent systems is the robust sharing of resources. The common solutions involve the serialization of access through mutual exclusion semaphores or queues. In object systems, such access control may be done through the Guarded Call Pattern or other design pattern approaches [5]. This can be indicated using the pattern notation of the UML or by adding an \{exclusion\} constraint on the relevant operation.

\subsection{Predictability}

A key aspect of many real-time systems is their predictability. This is crucial for many safety-critical and high-reliability systems, such as nuclear power plants, avionics systems, and medical devices. Predictability refers to the assurance that a system will always meet its timeliness requirements. This can be determined in some cases by static mathematical analysis, such as Rate Monotonic Analysis (RMA) [3]. In other cases, it can be ensured by disallowing preemption and using simple control algorithms, such as cyclic executives. Using active objects to represent tasks and identifying the performance characteristics of the tasks allows a variety of scheduling algorithms to be used.

Most real-time systems must be efficient both in terms of their timeliness as well as memory requirements. Object oriented systems can be just as efficient as structured systems. Object-oriented analysis allows, but does not require, better abstraction and encapsulation control. As with all modeling concepts, objectoriented or otherwise, it is up to the designer to select the appropriate level of abstraction to ensure correctness and maintainability while still meeting system performance requirements.

\subsection{Distributed Systems}

For large distributed multiprocessor systems, software components can be constructed. Components are run-time executable structures created from object models. These can be mapped to processor nodes using deployment diagrams. Deployment diagrams consist primarily of nodes (processors and other devices) 
linked together via interconnects. Selecting appropriate distribution architectures is commonly done by considering the tradeoffs of processor load balancing, communications bottlenecks, run-time flexibility via symmetric multiprocessing, and so on.

As mentioned above, communication among objects is modeled using the message abstraction. Although many systems can make do with commercial off-the-shelf (COTS) protocols, embedded systems must frequently use custom protocols to optimize the use of system resources, available bandwidth, and system reliability. Protocols can be, and are, profitably developed as a set of layered classes with elaborate state behavior to handle connections, disconnections, retries, timeouts, and failure modes. Object modeling is an ideal means for capturing the semantic details of complex communications protocols.

\subsection{Fault Tolerance and Safety}

Many embedded systems have high availability requirements. Typical applications include fire control, avionics, nuclear power, life support, and medical systems. Many of these systems must not only be reliable, but they must also be safe; that is, if they do fail, they do so without causing injury or loss of life.

There are many approaches to the development of reliable and safe systems, but all involve architectural redundancy in some form [3]. The common approach to capturing and representing this redundancy is through the use of architectural design patterns [5]. For example, the Heterogeneous Redundancy Pattern [ibid] arranges components into multiple redundant channels that can be used in either majority-wins-all or a failure-switchover policies. The Monitor-Actuator Pattern separates out the system actuation from the monitoring to ensure that single point failures can be identified and appropriate corrective action taken. Once again, objects provide a clear means for capturing and representing appropriate design solutions.

\subsection{Low-Level Hardware Interfacing}

Finally, a hallmark of real-time systems is the low-level control of hardware. Objects can easily and effectively model hardware interfaces [4]. Once modeled as classes, they may be subclassed and applied in a variety of environments. Once the interface to a low-level hardware device, such as an A/D converter, is modeled, then multiple instances of this class can directly represent the physical devices attached and controlled by your system.

\subsection{Modeling Real-Time Aspects of Systems}

In the UML, a constraint is a user-defined well-formedness rule that applies to one or more model elements. Development engineers have approached the problems of modeling the real-time aspects of systems is to apply constraints to specify the properties. The recently adopted $U M L^{T M}$ Profile for Schedulability, 
Performance, and Time Specification [2] (UPSPT) is an attempt to codify the standard ways that have been used now for several years of applying the UML to the problems of real-time and embedded systems. The profile really adds no new capabilities to the UML, but does standardize how certain aspects can be represented, facilitating model exchange.

Even though true profile, the UPSPT also provides metamodels for the various aspects related to schedulability, performance and time at least as a conceptual justification and explanation of the profile. The profile itself consists of a set of class stereotypes and various tagged values to specify various real-time properties. Since the tagged value identifiers are specified, this means that models can be exchanged among different kinds of tools for model entry, model analysis, code generation and so on.

\subsection{Using the UML in a Design Process}

The process described briefly below is the Rapid Object-Oriented Process for Embedded Systems (ROPES1 1 ). It has simultaneous three strong foci of concern:

- Architecture - which represents the large scale strategic decisions about the structure and behavior of the system as a whole

- Requirements - which capture both the functional and quality of service properties of the system required for correctness

- Models - which are used to represent the architecture and the requirements and ensure that they are consistent

The ROPES process is a spiral model, incrementally constructing the system throughout the development lifecycle. Figure 1 shows the primary process phases and their overall roles in product development.

Each spiral is divided into work phases, as shown in Figure 2 each of which has its own worker activities and artifacts.

Party Phase. This is also known as Process Planning Phase. The main activities here are

- Initial project planning (first iteration only)

- Review/assess/update

- Schedule

- Architecture

- Development Process

The primary artifacts produced and modified during this phase are the project schedule, architectural documents (normally class and object diagrams), and development plans.

\footnotetext{
${ }^{1}$ The ROPES process is discussed in more detail in 3]
} 


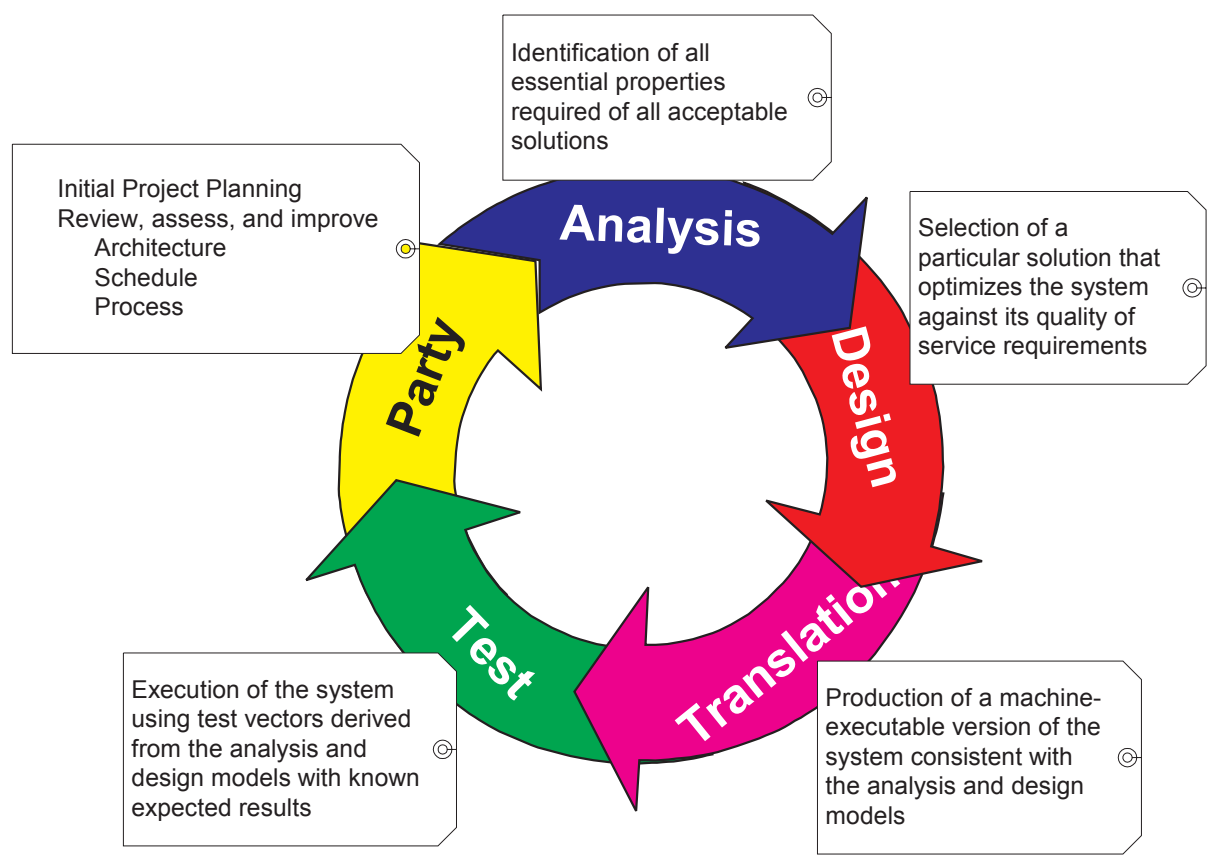

Fig. 1. Overview of Model-Driven Development

Analysis Phase. The analysis phase, as shown in Figure1, is all about identifying the essential properties true of all possibly acceptable solutions. The main activities are

- Requirements analysis - both functional and quality of service requirements are captured as use cases, use case specifications (such as with statecharts), and use case scenarios).

- Systems Engineering - this work activity is used only on larger systems, especially ones in which software and hardware is co-developed. The primary work activities here include the identification of the high-level subsystem architecture (captured with subsystem (object) diagrams), the interfaces among those, and the breakdown of responsibilities among the different disciplines contributing to the system (e.g. electronic, mechanical, chemical, and software). The architecture is tested early via execution of the subsystem model of the elaborated use case scenarios. This ensures that the architecture is adequate to support the responsibilities of the system to implement the captured requirements.

- Object Analysis - this work activity primarily identifies the software objects required to realize the use cases. These are represented via class and object diagrams, for the structural aspects, elaborated sequence diagrams (for the collaborative behavior), and statecharts (for the behavior of the individual objects). 


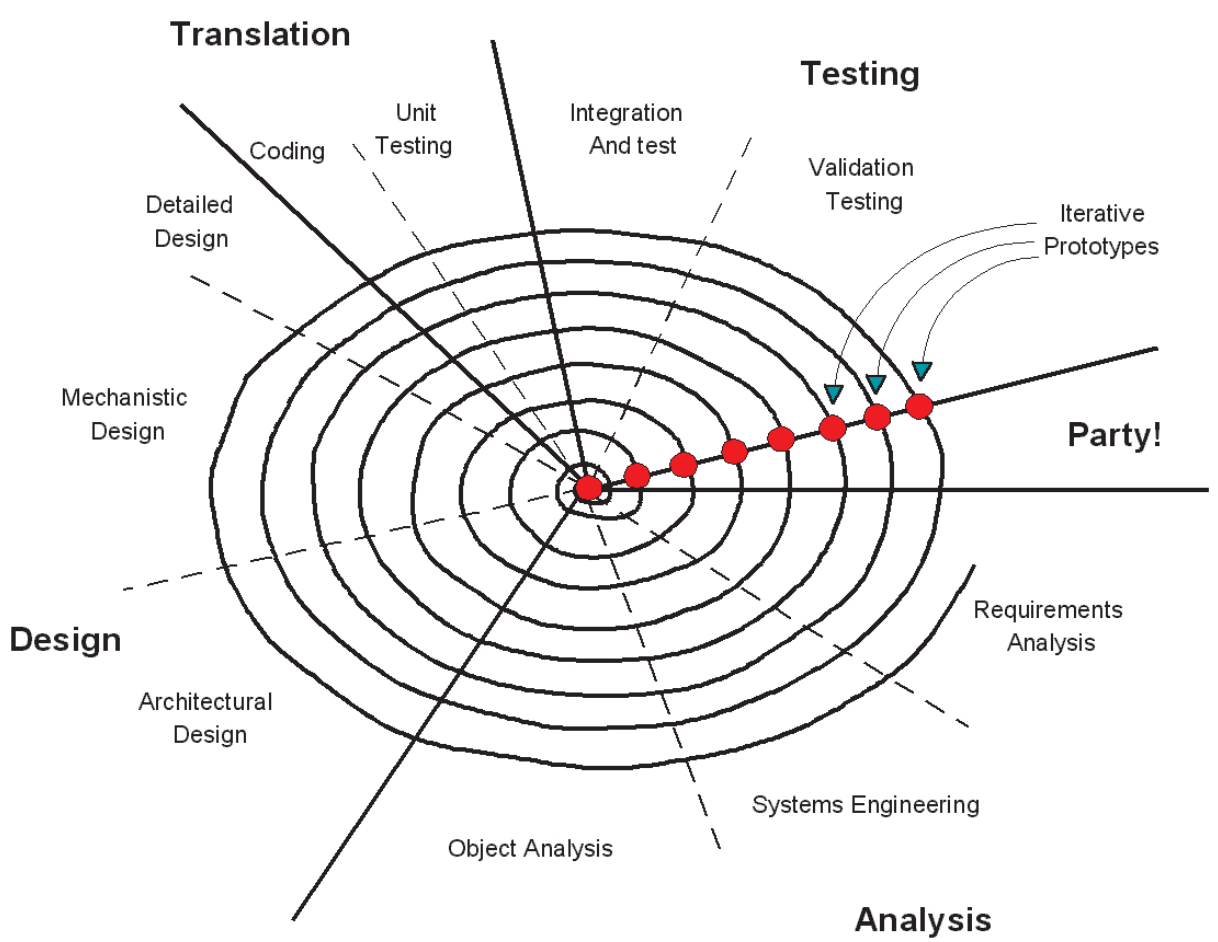

Fig. 2. ROPES Spiral (Detailed View)

Analysis is driven primarily by the functional requirements of the system.

Design Phase. The design phase picks a single solution that is consistent with the analysis model but is optimized or tuned for the particular product's optimality requirements. The design phase is all about optimization of the analysis model against all of the various quality of service requirements simultaneously. The primary subphases of design are:

- Architectqural Design - this subphase identifies the strategic design decisions that affect most or all of the system. There are 5 primary aspects of design which are identified in this phase captured using UML

- Subsystem/component architecture

- Concurrency architecture

- Distribution architecture

- Safety and reliability architecture

- Deployment architecture

- Mechanistic Design - this subphase optimizes individual collaborations that realize the use cases, so the scope of the design decisions are limited to the set of object realizing a specific use case. 
- Detailed Design - this subphase optimizes how the individual objects work, including their internal behavior with statecharts and activity diagrams, their internal data representation, and so on.

Design is driven primarily by the quality of service (QoS) requirements of the system.

Translation. The translation phase produces an executable version of the pieces of the system (components or subsystems) that will be integrated together in the testing phase. This means that the software can be generated from the models, either by hand, or automatically by a UML compiler, such as Rhapsody from I-Logix; the electronics can be wired together, perhaps as a breadboard, wirewrap, first-cut boards, or final boards. The primary subphases of translation are

- Implementation - the generation of the executable components of the system

- Unit Testing - the testing of the components or subsystems in isolation and then the peer review of the models and/or code of the tested component

Testing. The testing phase constructs the incremental prototype from the tested subsystems and components and tests it to ensure that the design is met (integration testing) and that it meets the requirements of the prototype (validation testing). The primary subphases are

- Integration Testing - construction of the incremental prototype from the tested subsystems and components, testing the interfaces among these large pieces of the system

- Validation Testing - application of the test vectors derived from the requirements in the requirements analysis phase to ensure that the prototype of the system meets its requirements.

It should be noted that "prototype" as used here means "executable version of the system" and NOT "throw-away proof-of-concept". In early spirals, the prototype will not have all of the requirements implemented nor will the ones implemented necessarily all be done to the ultimately required degree of fidelity. But as the system matures over time, it becomes more and more complete and correct, until one of the prototypes is the final released system.

\subsection{Requirements Capture with UML}

Use cases are the means that the UML provides to organize requirements. A use case organizes a cohesive set of requirements around a single (named) system capability but does not imply anything about internal implementation. A use case is represented on a use case diagram as a named oval, as shown in Figure 3 In this case, the system is an elevator and the use cases are the primary capabilities of the elevator. The stick figures in Figure 3 are called actors, and represent 


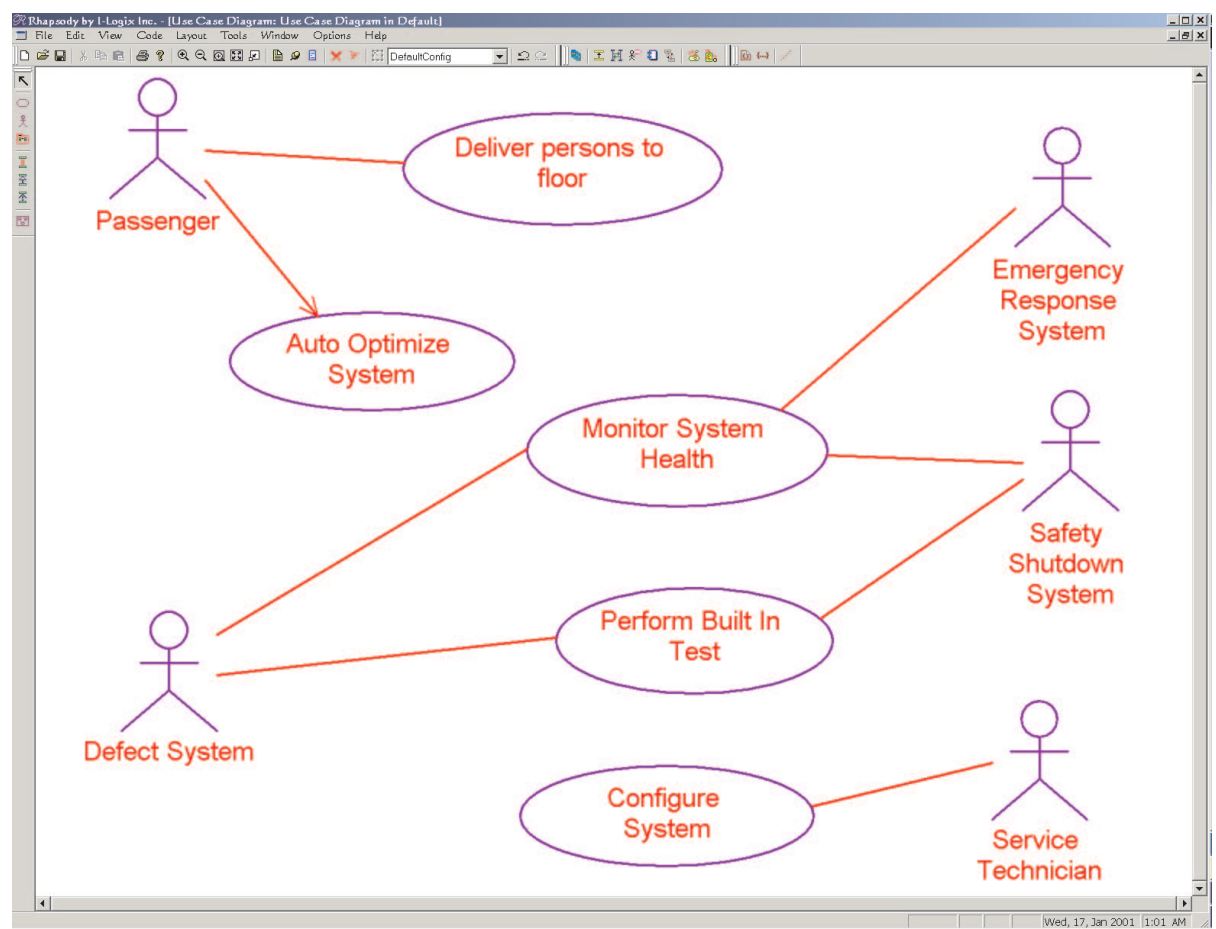

Fig. 3. Actors and Use Cases for an Elevator System

any object outside the scope of the system that interacts with the system in ways that we care about. The line connecting the actors to the use case means that that actor interacts in some interesting way with the system as the system executes that use case. You will notice in Figure 3, the association line connecting Passenger with Auto Optimize System is a directed line; this indicates that information flows in a single direction from the actor to the use case.

Use cases are named containers for more detailed requirements. What does "Monitor System Health" for the elevator system mean exactly? This use case internally contains more details about that it means. There are two primary means that the UML provides to what we call "detailing the requirements": namely, by example or by specification.

A common way to detail a use case is to provide a set of example scenarios. Each scenario captures a specific interaction of the use case (or, equivalently, the system executing the use case) with its associated actors. The use case is then detailed by providing a set of these scenarios, each scenario providing typical or exceptional cases of the use of that system capability. Scenarios capture 3 different kinds of requirements: messages or operations performed by the system, protocols of interaction between the system and its actors; and constraints on messages, operations, or protocols. 
A good thing about modeling scenarios is that non-technical stakeholders, such as managers, customer, and marketers, can easily understand how the system interacts with the actors in its environment in an implementation-free way. One downside of using scenarios is that there is an infinite set of scenarios; it may not always be obvious which scenarios ought to be modeled. In the ROPES process, a simple rule is used: each scenario ought to add at least one new requirement. Another downside is that scenarios provide only a means to model positive requirements, i.e. requirements that could be stated as "The system shall" but no way to model negative requirements, i.e. requirements that would be stated "The system shall not" Further, scenario model interactions between the system and its actors, but does not really provide a standard means to identify which interactions are required versus which may be incidental. For example, for an autopilot that can land an aircraft, the fact that landing gear are lowered prior to touching down is obviously crucial, but other messages may be order independent 2 .

Scenarios are modeled primarily with sequence diagrams, as shown in Figure 4. As is very common, the sequence diagram in Figure 4 describes the use case, its preconditions and postconditions, and keeps a running dialog explaining what's going on as the scenario unfolds. Note that how the system achieves the goals does not appear on the diagram, because that is the job for implementation and design, not for requirements capture.

This particular scenario shows what happens when P1 wants to go up and P2 wants to go down. Once can easily imagine many variants that show what happens when they both want to go up, what happens when the same request occurs several times, what happens when the cable breaks, and so on. Each of these different scenarios would be captured in a different sequence diagram, resulting in probably a dozen or so scenarios to elaborate what we mean by "Deliver Persons to Floor".

The other approach to detailing use cases is by specification. The UML provides statecharts and activity diagrams to specify what happens in all circumstances. Note that this is clearly different than sequence diagrams that show only a single example of actor-system interaction.

The statechart in Figure 5 specifies how the system responds to events. Most of the events come from the Passenger actor, of course, such as DownRequest, UpRequest, and FloorRequest. The actions specified on the statechart are the things that the system does in response to those incoming events, including sending messages back to the actors. For example, the GEN(Ack) action generates an acknowledgement that is sent back to the Passenger. The (c) symbols are conditional pseudostates and they represent branches taken based on the evaluation of guard conditions on out-going transitions, such as [Down Floor Request is queued].

The dashed lines represent concurrent regions of the statechart. Figure 5 has three such regions - one of which is responsible for the movement of the elevator,

\footnotetext{
${ }^{2}$ Most of the shortcomings of sequence diagrams are being addressed in the UML 2.0 specification.
} 


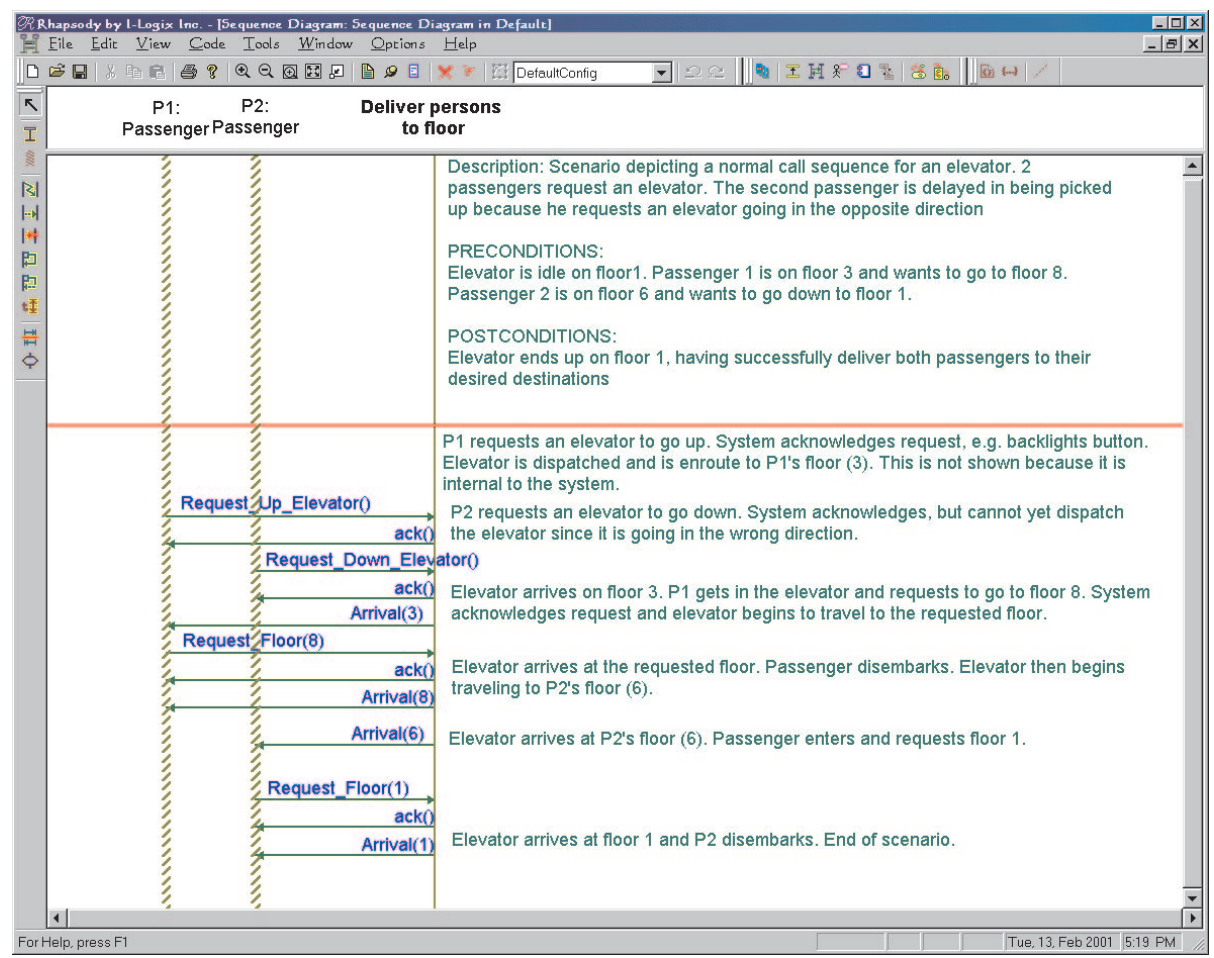

Fig. 4. Sequence Diagram

one responsible for handling incoming requests for an elevator going up or down, and one for handling requests to go to a specific floor once the passenger is in an elevator.

Because more people use the UML for systems design than for their requirements specification, it is actually more common to use statecharts and sequence diagrams to specify the behaviors of the internal structural pieces of the system. Nevertheless, they are useful for capturing, specifying, and illustrating requirements on the system as well.

\subsection{Design Structure with UML}

The UML is, at its core, an object oriented language based on the notion of objects and classes. An object is nothing more than one or more pieces of information tightly coupled together with operations that act on that information. An object occupies some particular location in memory for some specific period of time during the execution of the system. An object is an instance of a class. A class is nothing more than the specification of a set of objects. In the UML and other object-oriented languages, we design the structure of the system by identifying the objects, how they relate to each other, and their classes. 


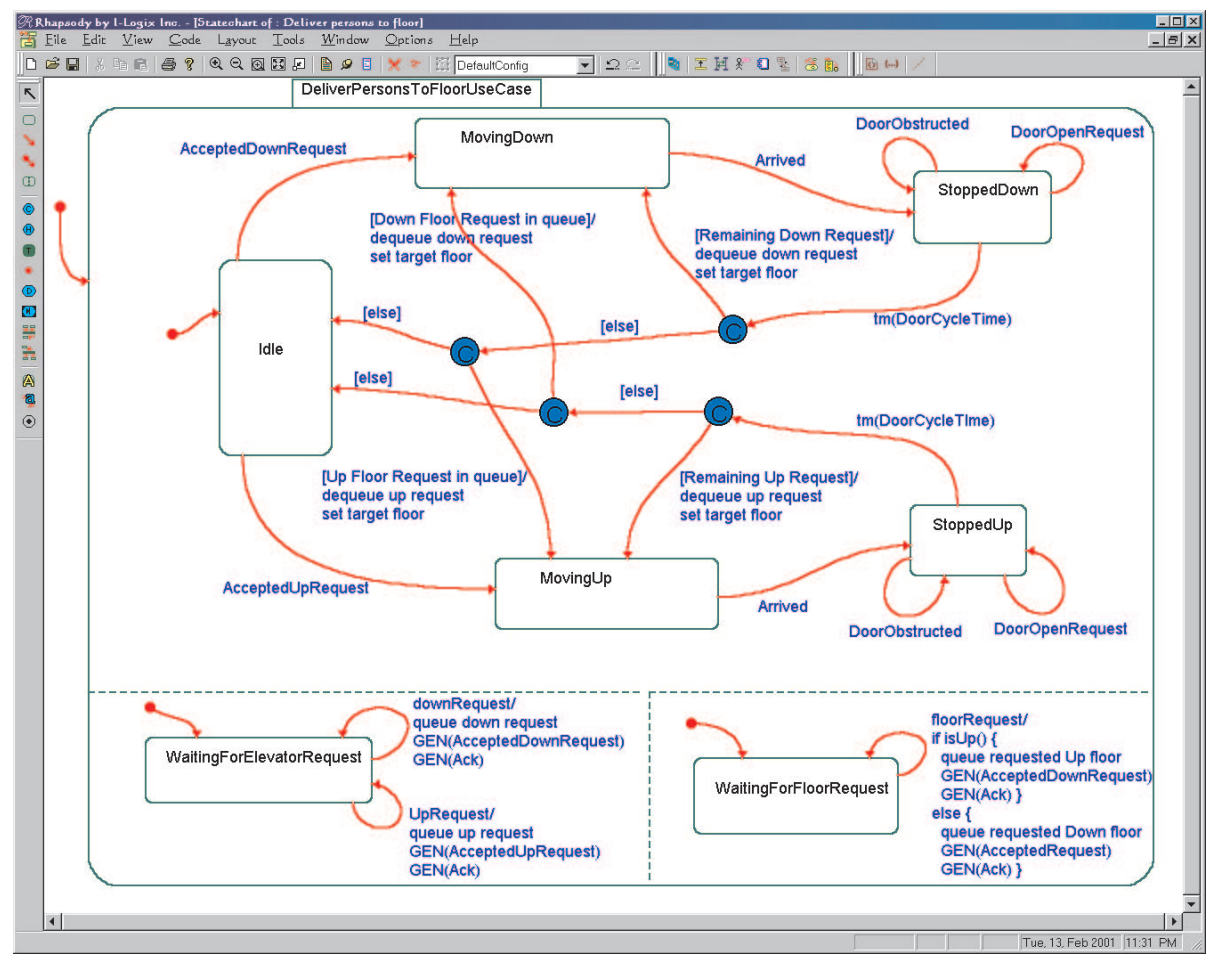

Fig. 5. Statechart for Elevator System

The UML is a graphical language, so diagrams are used to depict the object structure of the system. It is more common to show class diagrams, specifying the structure of collaborations of objects that will exist at run-time, rather than the object diagrams themselves. Such a class diagram for an embedded system is shown in Figure 6 .

The rectangles are the structural elements of the system - either classes (roughly, the "types" of the objects) or specific objects that exist in the system during execution. It is easy to tell the difference: if the name is underlined or contains a colon (as in UpButton : Button), then it is an object; otherwise it is a class, which represents one or more objects at run-time. Classes are typically shown either as simple named rectangles (such as backlight in the figure), as rectangles containing other rectangles (such as Elevator), or as a rectangle with multiple partitions (such as PositionSensor), depending on what you want to show about the class in question. The containment of one class within another is one way to show the composition relationship among classes. The multiple partition box allows you to show some or all of the attributes (information known by objects of the class) and some or all of the operations (behaviors which may be requested by the class). In the case of PositionSensor, the class has an attribute called position which is of type int, and it shows a single operation getPosition 


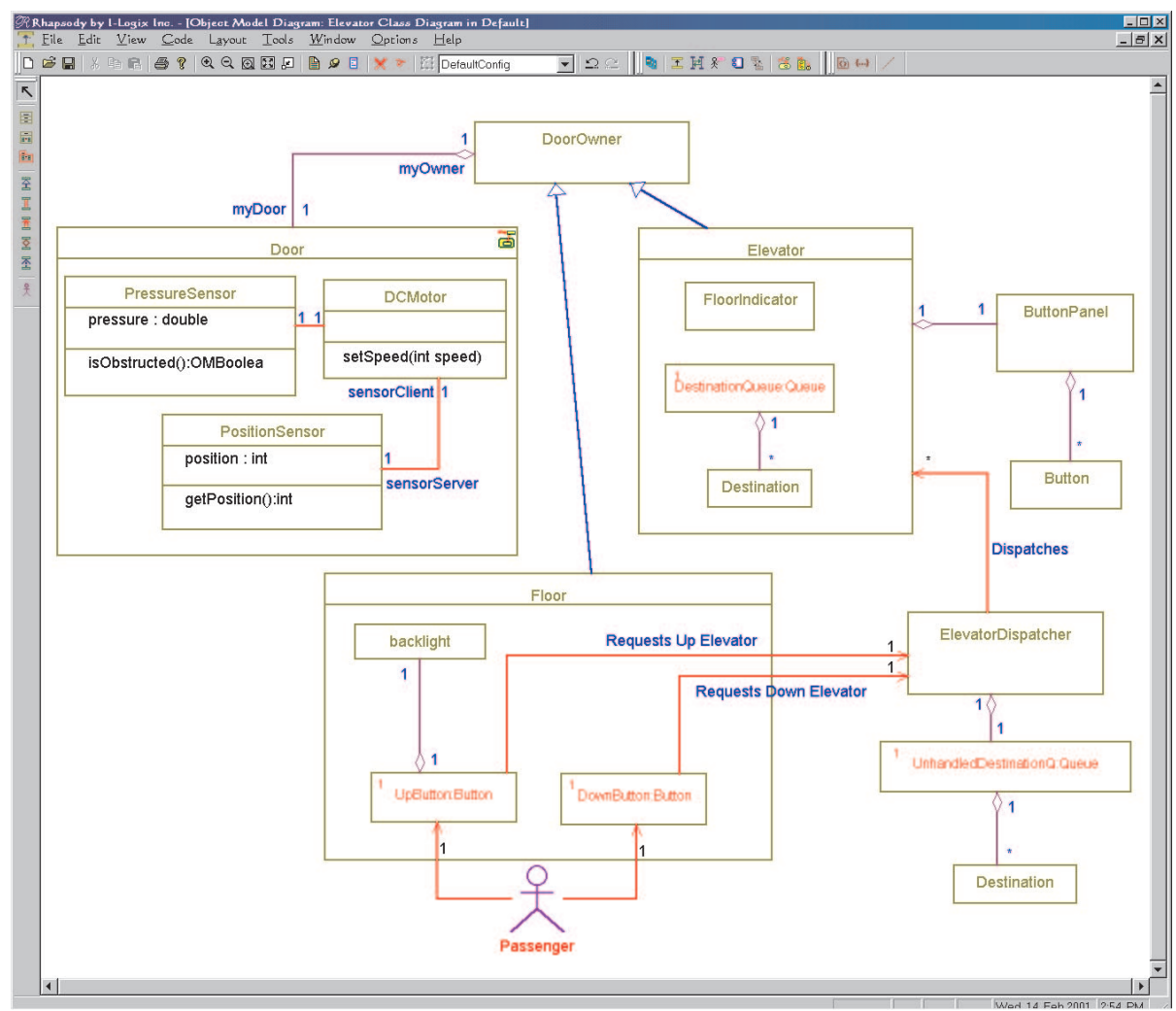

Fig. 6. Class Diagram for an Elevator System

which returns, unsurprisingly, an int. These different notations allow the designer to decide exactly how much he wishes to expose on any particular diagram. Note that PositionSensor may have additional attributes, such as perhaps a calibrationConstant and additional operations, such as executeSelfTest() but they are not shown on this diagram.

Most of the time, classes are shown on the diagram, but sometimes it is useful to show the named instances of those classes. For example, it is clear that the Floor object contains two objects of class Button, one called upButton and one called downButton. Both these objects are structurally the same and have the same set of operations since they are difference instances of the same class, but they serve different purposes in the executing system.

\subsection{Design Behavior with UML}

The other side of design, is of course, how the system behaviors, or acts over time. The UML provides ways of showing behavior of individual structural elements as well as how these elements behavior in the context of a collaboration. 
A structural element may behavior in one of three ways: simply, continuously, or reactively. Simple behavior means that how the object behaves is not dependent upon its history; for example, if I call PositionSensor.getPosition() it is likely to always be able to give me a valid response. The value will change depending on the position of the Door, but the kind of behavior executed remains the same. In continuous behavior, the behavior does depend on the object's history, but does so in a smooth way. For example, the DCMotor is likely to use control laws or differential equations to control the speed of the Door in a smooth fashion. The UML does not provide specific means for modeling continuous behavior; many developers find they have to go beyond the UML itself and use continuous mathematics-based tools to develop these algorithms. It is a simple matter to tie that behavior back into the structural model, however. The implementation of DCMotot.setSpeed () is provided by tools outside the standard UML and linked so that when that behavior is invoked, the separately-generated code is executed. Sometimes, both simple and continuous behavior are modeled with activity graphs.

Reactive behavior means that the object reacts to events (occurrences of interest that are sent to the object after they occur). The special thing about reactive behavior is that primarily the system waits for incoming events and then changes the kinds of things it does. Reactive behavior is modeled with finite state machines, hence the alternative name of stateful behavior.

The UML supplies rich finite state machine semantics via statecharts. Statecharts are an expanded notation with includes, as a subset, the traditional Mealy-Moore state machine semantics. A statechart is a state machine that supports additional constructs over the more traditional Mealy-Moore state machines, including:

- Nested states

- Or-states (exclusive)

- And-states (concurrent)

- History

- Conditional pseudostates (branching)

The statechart in the figure shows how the door behaves. We can see actions are executed either on the transition between states (such as the two actions executed on the OpenedEvent transition from the Opening::SlidingOpen state to the Open state, or on the entry into the state itself, such as the execution of the setInterlock(OFF) in the Closing::DisconnectingDoors state. Actions may also be executed when an object leaves a state as well. The transition with a single rounded end is called an initial state connector or an initial pseudostate. It indicates which is the default state when the object comes into existence or enters a state with substates.

The general form for a transition is

Event name '[' guard ']' '/' action list

Where 


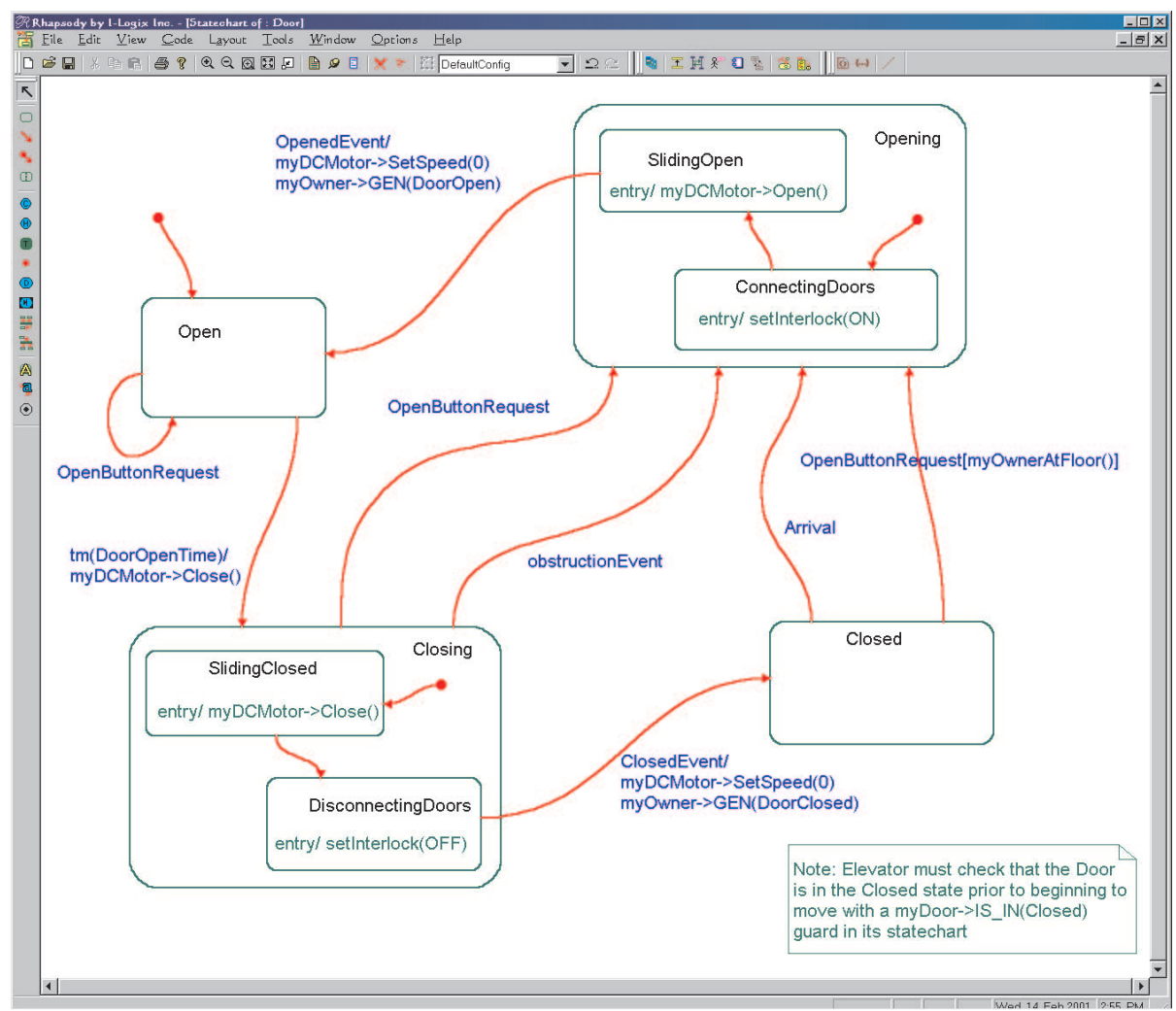

Fig. 7. Statechart for Door Class

- Event name is the name of the event received by the object that invokes the transition

- The guard is a Boolean expression. If present, the transition is only taken if the named event occurs and the guard evaluates to true

- The action list specifies a list of actions to be performed when the transition is taken. This can be primitive statements (such as $++\mathrm{x}$ ), operations on the object or other objects with which this object has an association, or the creation of an event (such as using the GEN() statement in the figure).

We can see how the Door processes the events that it receives. The Door starts off in the Open state. After a period of time (DoorOpenTime), a timeout fires and the Door transitions to its Closing State. The Closing state has two substates. The first (as indicated by the initial pseudostate) is the SlidingClosed State. While in this state, the Door commands the DCMotor to close the door. When that action is complete, the object transitions to the DisconnectingDoors state. If during the Closed state an obstruction is detected (as indicated by an event sent to the Door from the PressureSensor), then the Door transitions to the Opening State, and so on. 


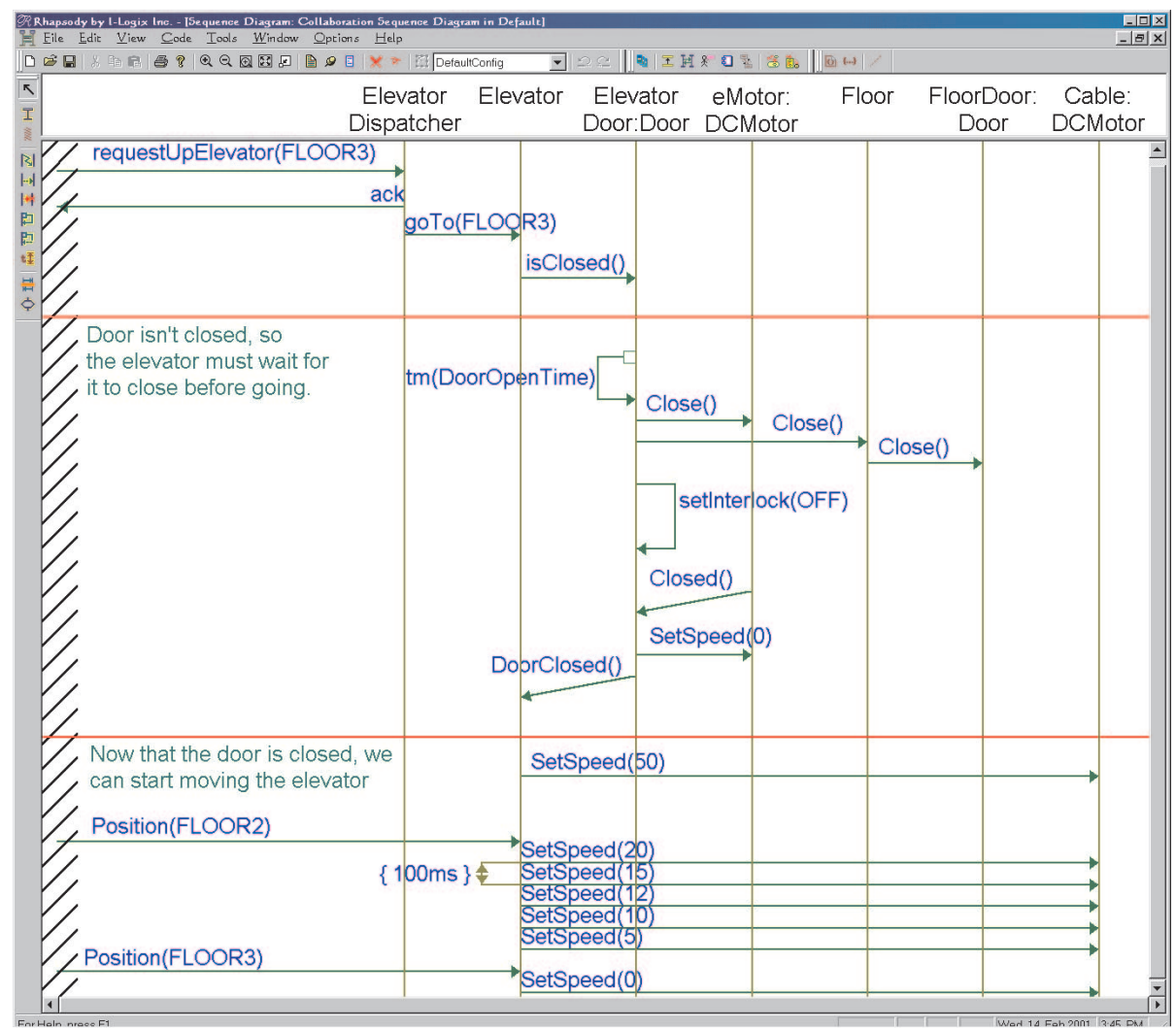

Fig. 8. Collaboration Sequence Diagram

Statecharts work very well for defining the behavior of reactive objects. But what if you want to understand how a set of objects work in collaboration? The UML provide sequence diagrams to show how object collaborate by sending message (calling operations) and events to each other.

Figure 8 shows an illustrative example of a sequence diagram. The vertical lines represent objects (not classes) during the execution of the system. The arrowed lines are messages (either calls to operations on objects or events sent to objects). The hatched area on the left is called a "collaboration boundary" and represents all objects in the universe other than those explicitly represented as instance lines on the diagram. Time flows down the page. It is easy to see how these objects work together to produce the desired system effect - picking up and delivering a person, upon request, from one floor to another. The scenario isn't complete, but nevertheless it shows most of the major elements of sequence diagrams: instance lines, messages, events, comments, and even a timing constraint (the number in curly braces is expressing the time between two message invocations). Although you can see very easily how the objects collaborate together, the internal behavior of individual objects is not readily apparent. 


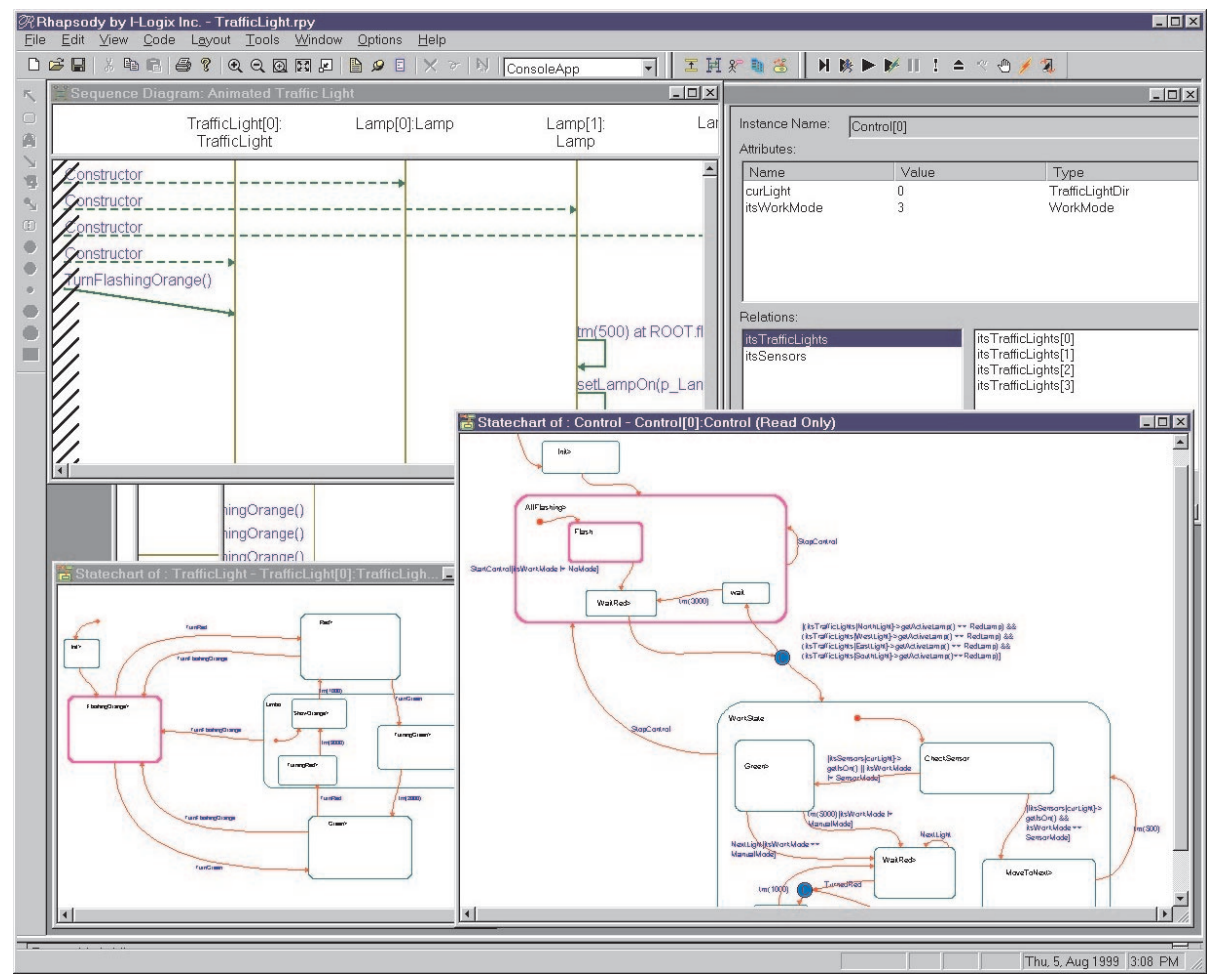

Fig. 9. Executing the UML Model

\subsection{Executing UML Models}

As one of the early pioneers of computers, Immanuel Kant, said in the 17th century, modeling has a fundamental problem which he called the "analyticsynthetic dichotomy". Simply put, is states "that which is real (i.e. running) cannot be known, and that which is known (i.e. modeled) cannot be real." In most modeling languages, that is more or less true, because the models only bear a vague resemblance to the executing system. With UML, however, the core of the behavioral model is based on statecharts, a well-understood executable technology. With the UML, it is possible to execute your models and to use them to synthesize your software (and potentially hardware as well).

It has been said that the best way not to have bugs in your delivered system is not to put them in the system in the first place. Since no ones knows how to do that yet, the next best thing is to remove them immediately after you put them in. How do you do that? Simple - you execute your model early and often.

A snapshot of an executing UML is shown in the last figure 1]. Statecharts are color coded to show the current state of the object, while a sequence diagram is dynamically drawn as the objects collaborate during the execution of the system. Additionally, the current values of the attributes can be shown. 
The ability to execute even partial UML models means that we can test our models for correctness as early as possible and as often as we want with very little effort (other than pushing the "Compile and Run" button). Eliminating the need to manual write source code for the models means that the model and the code are always in synch, solving Kant's analytic-synthetic dichotomy. It also removes developer coding defects, thereby improving system quality and reducing time-to-market.

\section{References}

1. Screenshot of Rhapsody, an executable UML modeling and development tool. For information see www.ilogix.com.

2. $\mathrm{UML}^{\mathrm{TM}}$ profile for schedulability, performance, and time specification. OMG document ptc/02-03-02, Object Management Group 2002.

3. Bruce Powel Douglass. Doing hard time: Developing real-time systems with UML. Addison Wesley, 1999.

4. Bruce Powel Douglass. Real-Time UML $2^{\text {nd }}$ Edition: Developing Efficient Objects for Embedded Systems. Addison Wesley, 1999.

5. Bruce Powel Douglass. Real-Time Design Patterns: Robust scalable architecture for real-time systems. Addison Wesley, 2003. in press. 